\title{
Analizando los esfuerzos para la conservación de carnívoros terrestres en Argentina
}

\section{Analyzing efforts for the conservation of the terrestrial carnivores of Argentina}

\author{
Mauro Lucherini, ${ }^{1,5 *}$, Juan I. Reppucci ${ }^{1,2,5}$, Lucía Soler ${ }^{1}$, Alejandro González ${ }^{3}$, Paula González \\ Ciccia ${ }^{4}$, Rocio Palacios ${ }^{5}$, Javier A. Pereira ${ }^{6}$ \& Sonia Zapata ${ }^{7}$
}

${ }^{1}$ Grupo de Ecología Comportamental de Mamíferos (GECM), Laboratorio de Fisiología Animal, Instituto de Ciencias Biológicas y Biomédicas del Sur (INBIOSUR), Departamento de Biología Bioquímica y Farmacia, Universidad Nacional del Sur (UNS), Consejo Nacional de Investigaciones Científicas y Técnicas (CONICET), Bahía Blanca, Argentina.

2Delegación Regional Noroeste, Administración de Parques Nacionales, Consejo Nacional de Investigaciones Científicas y Técnicas (CONICET), Salta, Argentina.

${ }^{3}$ Dirección de Ecosistemas Terrestres, Centro de Ecología Aplicada del Neuquén, Junín de los Andes, Neuquén, Argentina. ¿Unidad de Gestión estratégica y Transformación. EPI. Gobierno de la Ciudad de Buenos Aires”, Ciudad Autónoma de Buenos Aires, Argentina.

${ }^{5}$ Alianza Gato Andino. Villa Carlos Paz, Córdoba, Argentina.

${ }^{6}$ División Mastozoología, Museo Argentino de Ciencias Naturales "Bernardino Rivadavia" Consejo Nacional de Investigaciones Científicas y Técnicas (CONICET), Ciudad Autónoma de Buenos Aires, Argentina.

${ }^{7}$ Centro de Investigaciones de Puerto Deseado-ICASUR, Universidad Nacional de la Patagonia Austral, Puerto Deseado, Santa Cruz, Argentina.

*E-mail: lucherinima@yahoo.com

\section{RESUMEN}

Debido a la gran diversidad (28 especies) y el delicado estado de conservación $(57,1 \%$ de especies con amenaza de extinción) de los carnívoros terrestres en Argentina, nos propusimos recopilar información sobre los esfuerzos actuales de conservación en este país y, mediante consulta a expertos, identificar medidas para mejorar su eficacia. El principal obstáculo encontrado para la implementación de proyectos de conservación en Argentina fue la falta de interés por parte de entes gubernamentales, aunque este factor habría evolucionado positivamente en los últimos 5 años. La limitada participación de las comunidades locales y la escasez de fondos fueron otros problemas importantes y con tendencia variable entre proyectos. Las carencias en el sistema educativo y de enfoques interdisciplinarios representarían obstáculos menos relevantes. Se registraron 29 proyectos de conservación de los carnívoros en desarrollo en 19 de las 23 provincias argentinas, con mayor concentración en Neuquén y provincias del norte del país. El 78,6\% de las especies de carnívoros nativos es objeto de uno o más proyectos, pero menos de la mitad son el foco de un proyecto. El número de proyectos por especie varía entre 16 (para Puma concolor) y cero (para Pteronura brasiliensis, Lontra felina, Lyncodon patagonicus, Conepatus humboldtii y Leopardus guigna). La cantidad de proyectos por especie no resultó relacionada con su estado de conservación, pero sí con su tamaño corporal. Se brindan pautas para corregir sesgos y establecer prioridades taxonómicas y geográficas y así mejorar el uso de los limitados recursos disponibles para la conservación de los carnívoros argentinos.

Palabras clave: Canidae, Felidae. Mustelidae, Priorización, Procyonidae.

\begin{abstract}
Because of the great diversity (28 species) and fragile conservation status (57.1\% of species are in threat of extinction) of the terrestrial carnivores of Argentina, we aimed to collect information about the conservation efforts in this country and identify tools to improve their effectiveness through expert opinion. Lack of interest by governmental agencies was the major obstacle for the development of conservation projects in Argentina. However, this factor appears to have evolved positively in the last 5 years. The limited participation of local communities and lack of funds were recognized as additional impediments, which showed variable temporal trends. The inadequacies of the education system and the lack of interdisciplinary approaches represented less important difficulties. Twenty-nine current carnivore conservation projects were recorded in 19 of the 23 Argentinean provinces, with a concentration in Neuquén and some northern provinces.
\end{abstract}


Although 78.6\% of the species of native carnivores forms part of at least one project, less than half of them are the focal species of a project. The number of projects per species ranges from 16 (for Puma concolor) to cero (for Pteronura brasiliensis, Lontra felina, Lyncodon patagonicus, Conepatus humboldtii, and Leopardus guigna). Whereas the number of projects/species was not related to a species' conservation status, we found a positive association between this indicator of conservation effort and body size. We provide suggestions to reduce biases and identify taxonomic and geographic priorities that will enable to improve the use of the limited resources available for the conservation of carnivores in Argentina.

KeYwords: Canidae, Felidae, Mustelidae, Prioritization, Procyonidae.

\section{INTRODUCCIÓN}

Los carnívoros (Mammalia: Carnivora) son uno de los taxones de animales más carismáticos y amenazados (Ray 2005; Karanth \& Chellam 2009). Por estas mismas razones, y por algunas características biológicas subyacentes, la conservación de los carnívoros es considerada de gran importancia (Ripple et al. 2014) y éstos son frecuentemente utilizados como especies focales o bandera en programas y estrategias de conservación de la biodiversidad (Ginsberg 2001; Sergio et al. 2008).

De todos los mamíferos terrestres del país los carnívoros son el segundo grupo (después de los ungulados) en términos de proporción de especies amenazadas sobre el total de especies evaluadas (Fig. 1), con un incremento del 7,1\% con respecto a la revisión anterior del estado de conservación (Díaz \& Ojeda 2000). A la llegada de los europeos, el territorio que actualmente corresponde a la República Argentina albergaba 29 especies de carnívoros terrestres (se excluyen las Familias Otariidae y Phocidae), aproximadamente el $46 \%$ de todos los carnívoros registrados para América Latina. Una de ellas, el zorro de las Malvinas (Dusicyon australis, Kerr 1792), se encuentra extinta. Según el Libro Rojo de Mamíferos Amenazados de

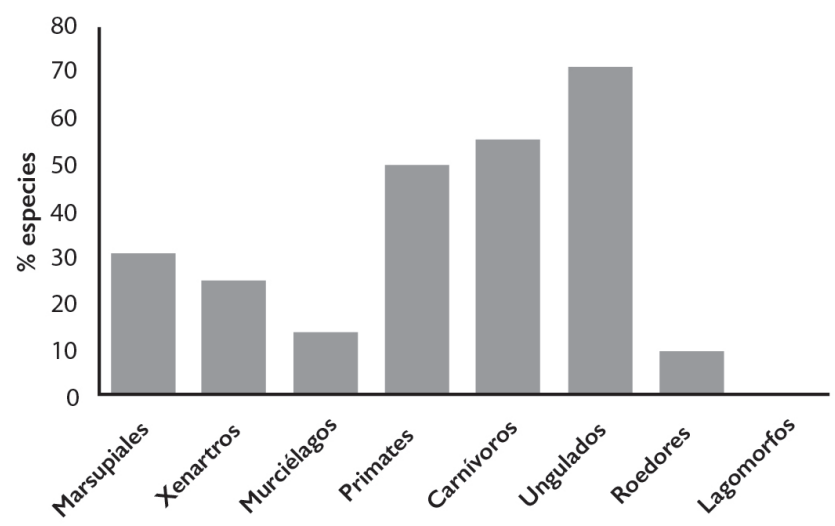

Figura 1. Proporción de especies amenazadas en cada Orden de mamíferos argentinos, sobre la base de la categorización de Ojeda et al. (2012). / Proportion of threatened species for each Argentinean mammal Order, based on Ojeda et al. (2012) categorization. la Argentina (Ojeda et al. 2012), 15 especies de carnívoros $(57,1 \%)$ recaen en alguna de las categorías de amenaza reconocidas por la Unión Internacional para la Conservación de la Naturaleza (UICN), 12 no se encuentran amenazadas y completan la lista una especie para la cual se considera que no hay datos suficientes para la categorización de su estado de conservación y una que no fue evaluada (Fig. 2). Si los mefítidos son incluidos en la Familia Mustelidae para facilitar la comparación con la revisión de Díaz \& Ojeda (2000), el porcentaje de especies amenazadas es particularmente alto en las Familias Felidae (70\%) y Mustelidae (66,7\%); mientras que la Familia Canidae es la que presenta la mayor proporción de especies no amenazadas (66,7\%, Fig. 2). La pérdida y degradación de hábitat y la caza serían los principales factores que explican la reducción de sus poblaciones (Ojeda et al. 2012). A pesar de la gran diversidad y de la delicada situación de conservación de los carnívoros en Argentina, hasta la década pasada el conocimiento sobre la mayoría de estas especies era escaso (como sugiere un estudio sobre la Familia Felidae; Lucherini et al. 2004) y pocos eran los proyectos dedicados a su conservación. La falta de información representa un claro obstáculo en la toma de decisiones para enfrentar los problemas de conservación.

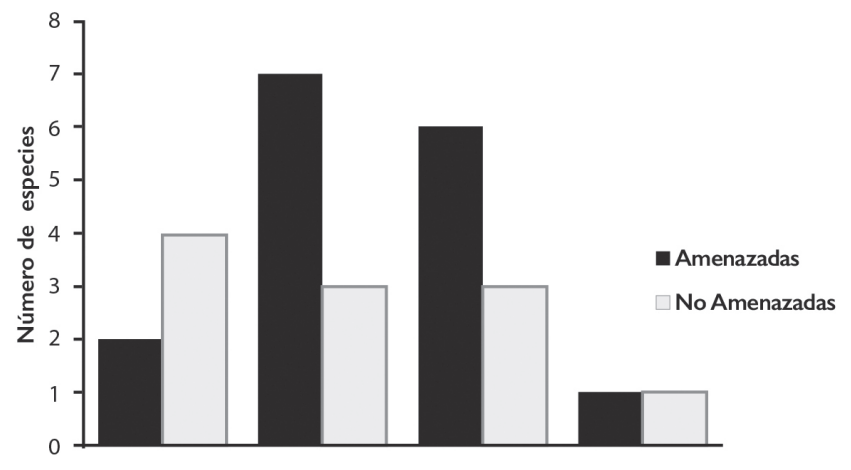

Figura 2. Cantidad de especies amenazadas y no amenazadas en cada Familia de carnívoros terrestres argentinos, sobre la base de la categorización de Ojeda et al. (2012). Los mefítidos quedaron incluidos en la Familia Mustelidae. / Number of threatened and unthreatened species for each terrestrial carnivore Family in Argentina, based on Ojeda et al. (2012) categorization. Mephitids were included in the Mustelidae Family. 
Con el objetivo de identificar los factores que limitaron los esfuerzos para la conservación de los carnívoros terrestres en la última decena de años en Argentina, en noviembre de 2010 se realizó un taller en el marco de las XXIII Jornadas de la Sociedad Argentina para el Estudio de los Mamíferos (SAREM; Apéndice I). El presente trabajo se propone retomar los resultados de ese encuentro, actualizarlos y llevar a cabo una evaluación de los esfuerzos para la conservación de los carnívoros terrestres en Argentina. Los objetivos específicos son: 1. Analizar cómo los proyectos de conservación de carnívoros se distribuyen geográficamente y con respecto al estatus de conservación de las especies. Debido a que el tamaño corporal puede influir sobre los esfuerzos de investigación de carnívoros (Brooke et al. 2014) y la probabilidad que una especie sea legalmente protegida (Metrick \& Weitzman 1998), analizamos además si los proyectos de conservación de carnívoros en Argentina se distribuyen homogéneamente con respecto a este factor. 2. identificar los mayores obstáculos para la implementación de proyectos de conservación de carnívoros en Argentina, evaluando en qué forma estas limitaciones han cambiado en el periodo 2010-2014. 3. Proponer medidas que permitirían mejorar la eficacia de estos proyectos.

\section{MÉTODOS}

El conocimiento de expertos es una fuente de información cuyo uso se está incrementando en ecología, especialmente en revisiones asociadas con la conservación de la biodiversidad (Clevenger et al. 2002; Regan et al. 2004; Boitani et al. 2007; Martin et al. 2012) y es la principal herramienta que se utilizó en este trabajo.

La base de información para este trabajo se recolectó durante el taller "Análisis de estrategias para la conservación de carnívoros en Latinoamérica" (en adelante "Taller") realizado en el marco de las XXIII Jornadas de SAREM (Apéndice I). Todos los participantes respondieron, antes de las Jornadas, unas preguntas que apuntaban a identificar las principales dificultades enfrentadas en sus actividades de conservación de carnívoros, así como los desafíos para mejorar la efectividad de sus proyectos. Cada participante realizó una presentación durante el Taller en la que describió su proyecto y posteriormente se acordó, entre todos los participantes, las mayores dificultades encontradas y las causas de las mismas.

A la luz de la publicación del nuevo Libro Rojo de Mamíferos Amenazados de la Argentina (Ojeda et al. 2012), cuatro años más tarde los autores acordaron retomar los resultados obtenidos en el mismo, completarlos y actualizarlos para responder a los objetivos propuestos en este trabajo. Con este propósito, se ha realizado un sondeo que trató de recopilar la información básica (ubicación geográfica, responsables, especies focales y secundarias) de todos los proyectos de conservación in-situ de carnívoros terrestres actualmente en curso en Argentina. Los criterios de base para incluir un proyecto sobre carnívoros dentro del análisis de esta publicación fueron: 1. que tuviera uno o más objetivos de conservación claramente explícitos y 2. que estuviera implementando acciones para cumplir con esos objetivos. Para llegar a la lista final se usó inicialmente la técnica de la bola de nieve ("snow-ball"; Bailey 1982), solicitando a cada autor y a colaboradores que mencionaran los proyectos que, en su opinión, cumplían con los criterios mencionados y después haciendo lo mismo con los investigadores que fueron identificados como los referentes de esos proyectos. De esta forma el número de proyectos incluidos fue aumentando hasta llegar a una asíntota horizontal. Finalmente se hizo circular entre los autores una lista de los proyectos para lograr un consenso general sobre su representatividad.

Con el objetivo de analizar las relaciones entre los esfuerzos de estudio y conservación de los carnívoros terrestres argentinos, descritos en la lista de proyectos y el estatus de conservación de los mismos (ver Apéndice II), se utilizó la categorización del estado de conservación asignada a cada especie en Ojeda et al. (2012), basada en las categorías de amenaza de la Unión Internacional para la Conservación de la Naturaleza (UICN). Para este análisis, las especies categorizadas como en peligro crítico (CR) y en peligro (EN) fueron reunidas en una nueva categoría definida de "prioridad alta" de conservación, las especies en la categoría vulnerables (VU) fueron definidas de "prioridad media" y aquellas clasificadas como casi amenazadas (NT) y de preocupación menor (LC) fueron agrupadas en la categoría "prioridad baja". Para analizar la relación entre el tamaño corporal de las especies y los esfuerzos dedicados a ellas asignamos las especies a tres categorías en función de su peso corporal (grande, cuando su peso promedio > $20 \mathrm{~kg}$; mediano, peso promedio entre 6 y $20 \mathrm{~kg}$; pequeño, peso promedio $<6 \mathrm{~kg}$ ). Los pesos corporales se obtuvieron de Canevari y Vaccaro (2007), a excepción de Lyncodon patagonicus (de Blainville 1842), para el cual usamos Chebez et al. (2014).

Finalmente, para determinar la relevancia y las tendencias con respecto a las dificultades y desafíos que conlleva el trabajo de conservación de carnívoros terrestres en Argentina, se solicitó en forma individual a todos los autores que priorizaran las dificultades identificadas en el taller y evaluaran su cambio en los últimos cinco años. Con el propósito de asignar el nivel de prioridad de los obstáculos se utilizaron tres categorías (Fundamental, Importante, Secundario), mientras que para evaluar si hubo cambios en los últimos 5 años se adoptaron las categorías "Mejora", "Ningún Cambio", "Empeoramiento". 


\section{RESULTADOS}

Las principales dificultades encontradas en la implementación de proyectos de conservación por los participantes del Taller fueron:

- Escasez DE Fondos. La obtención de subsidios es dificultosa, por lo cual los investigadores deben invertir mucha energía y esfuerzo para lograr financiación suficiente, en lugar que dedicarse a la implementación de proyectos.

- Escasa PARTICIPACIÓN DE LAS COMUNIDADES LOCALES. Las razones del escaso involucramiento por parte de las comunidades locales en los problemas y los proyectos de conservación que se desarrollan en sus áreas son diferentes, pero en muchos casos están relacionadas a la falta de una estrategia específica por parte de los líderes de los proyectos de conservación. Independientemente de la causa, este obstáculo dificulta lograr objetivos de conservación a largo plazo y que las actividades de conservación sean adoptadas por parte de las comunidades locales.

- Carencias en la función docente en relación con la conservación de la biodiversidad. Los docentes, en particular en escuelas rurales, en muchos casos por falta de capacitación específica, no cumplen una función como agentes multiplicadores de las temáticas de conservación. Esta carencia en el sistema educativo, a su vez, limita la posibilidad que se cree una mayor conciencia ambiental, lo cual afecta el éxito de estrategias de conservación de la biodiversidad, particularmente en el caso de los carnívoros, que son frecuentemente objetos de conflictos con el hombre (Treves \& Karanth 2003).

- INSUFICIENCIA DE ENFOQUES INTERDISCIPLINARIOS. Si bien sería deseable que los biólogos trabajaran en conjunto con sociólogos, antropólogos, geógrafos y economistas, entre otros profesionales, los grupos de investigación raramente establecen colaboraciones interdisciplinarias.
- FALTA GENERALIZADA DE INTERÉS DE LAS DISTINTAS ENTIDADES DEL GOBIERNO en la conservación de la biodiversidad. Este problema puede darse en la instancia de la toma de decisiones legislativas y/o en la implementación de las mismas y abarca varios aspectos, entre los cuales se pueden mencionar: la escasa participación en proyectos en conjunto con otras instituciones de parte de los representantes del gobierno; la falta de políticas de desarrollo económico sustentables (que incrementa la problemática en la conservación de la biodiversidad en general); la escasez de cargos ocupados por personal idóneo en las entidades del gobierno con injerencia en manejo de los recursos naturales; los frecuentes cambios de gobiernos, que dificultan la planificación a largo plazo.

El 75\% de los autores identificó a la falta de interés por parte del gobierno como un obstáculo fundamental para la implementación de proyectos de conservación de carnívoros en Argentina, poniendo este tema en la cima de la lista de las dificultades (Fig. 3). A pesar de la gravedad con la cual fue percibida, la falta de interés de las instituciones gubernamentales se estaría incrementando sólo para el 25\% de los autores, mientras que el 50\% de los mismos notó una evolución positiva, lo cual coloca este tema por debajo del promedio de los valores del análisis de tendencia (Fig. 4). La poca participación de las comunidades locales y la escasez de fondos fueron los otros problemas cuyos valores de importancia relativa superaron el valor promedio y fueron indicados como "fundamentales" por el 50\% y el 38\% de los autores, respectivamente (Fig. 3). Sin embargo, con respecto a la escasez de fondos se registró un equilibrio entre los que consideraron que este problema se está reduciendo y aquellos que notaron su incremento (calificándose este tema como el de tendencia más negativa), mientras que la participación local calificó como un problema que estaría

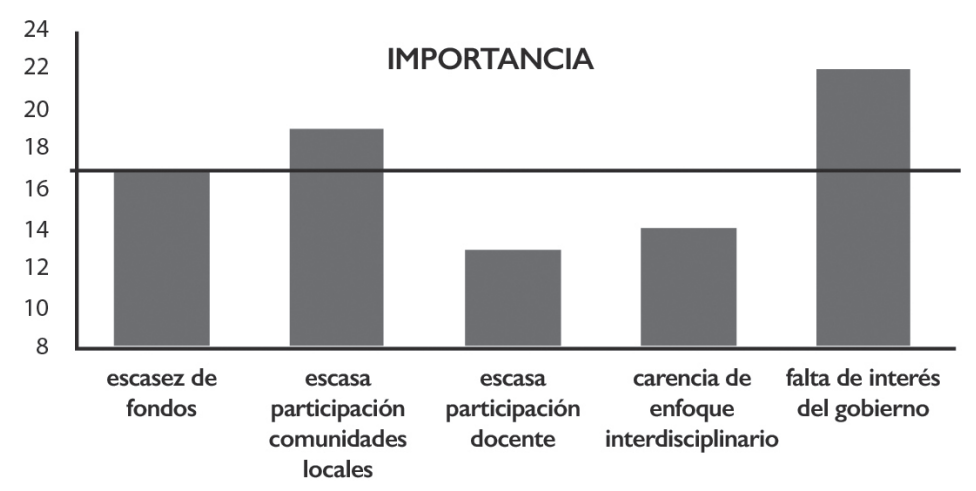

FIGURA 3. Importancia relativa de las principales dificultades para la implementación de proyecto de conservación de carnívoros terrestres en Argentina según la opinión de los autores. El valor del índice puede variar entre 8, si todos los encuestados asignaran una importancia escasa a la dificultad, y 24, si todos los encuestados consideraran ese factor fundamental. La línea negra indica el valor promedio. / Relative importance of the major obstacles in the implementation of conservation projects on terrestrial carnivores in Argentina, based on the authors' opinions. The value of the index may vary from 8 when all authors assigned a reduced relevance to a given obstacle, to 24 when all authors considered that obstacle as fundamental. The black line shows the average value. 
reduciéndose para el 50\% de los autores y que no mostró cambios para el restante $50 \%$ (Fig. 4). Las carencias en el sistema educativo, al contrario, fueron consideradas de importancia secundaria por el $66,7 \%$ (Fig. 3) y estarían mostrando una mejoría para el 33\% y ningún cambio de tendencia para el $50 \%$ de los autores que las mencionaron (Fig. 4). La carencia de un enfoque interdisciplinario en los proyectos de conservación no representaría un obstáculo importante (solo el $25 \%$ de los autores lo clasificó como "fundamental"; Fig. 3) y tendría una tendencia relativamente positiva (Fig. 4). Cabe mencionar que ninguno de los problemas presentaría una tendencia claramente negativa en los últimos 5 años (Fig. 4).

Nuestro sondeo registró 29 proyectos que se proponen apoyar de alguna forma la conservación de los carnívoros terrestres argentinos (Apéndice II). Todos los proyectos incluyen por lo menos un/a argentino/a entre los Investigadores Principales. Éstos son 65 en total (2,24 por proyecto, en promedio), de los cuales 20 están involucrados en 2 o más proyectos. En 19 de las 23 provincias argentinas se están desarrollando uno o más proyectos de investigación. Si bien el promedio es 2,26 proyectos/ provincia, la distribución geográfica no es uniforme y hay una concentración de proyectos en algunas provincias (Fig. 5). El 78,6\% de las especies de carnívoros terrestres de Argentina es objeto de por lo menos uno de estos proyectos. Sin embargo, esta proporción baja al $46,4 \%$ si se consideran únicamente las especies que han sido identificadas como focales para los proyectos. En forma similar a la distribución geográfica de los proyectos, aun cuando hay un promedio de 3,19 proyectos por especie, la distribución de los proyectos entre las especies no es homogénea (Fig. 6). El rango del número de proyectos por especie varía entre 16, en el caso de Puma concolor (Linnaeus 1771) y cero (para Pteronura brasiliensis (Gmelin 1778), Lontra felina (Molina 1782), L. patagonicus, Conepatus humboldtii (Gray 1837) y Leopardus guigna (Molina 1782). Este patrón se repite para las especies focales de los proyectos, donde se destacan P. concolor con 13 proyectos y Panthera onca (Linneus 1758) con 9, mientras que hay 13 especies que, si bien están listadas en algún proyecto, no son el foco de ninguno de ellos (Eira barbara (Linneus 1758), Galictis cuja (Molina 1782), Conepatus chinga (Molina 1782), Puma (Herpailurus) yagouaroundi (Geoffroy 1803), Leopardus colocolo (Molina 1782), Cerdocyon thous (Linneus 1766), Procyon cancrivorus (Cuvier 1798) y Nasua nasua (Linneus 1766). En promedio, las 9 especies con prioridad alta de conservación son objeto de 2,1 proyectos/especie, aquellas de prioridad media $(\mathrm{N}=6)$ de 3,3 proyectos, mientras que las 12 especies de prioridad baja de conservación fueron incluidas en 3,9 proyectos. Sin embargo, las especies de prioridad alta fueron indicadas como especies focales en 16 proyectos (1,8 proyectos, en promedio), las de prioridad media en 7 proyectos (promedio: 1,2 proyectos) y las de prioridad baja son especies focales en 22 proyectos, con un promedio de 1,8 proyectos/especie.

Se observó una relación fuerte entre el esfuerzo de conservación y el tamaño corporal de las especies involucradas en los proyectos $\left(\mathrm{R}^{2}=0,59, \mathrm{p}<0,001\right)$. El número de proyectos/especie es considerablemente más alto para los grandes carnívoros que las especies de tamaño mediano y pequeño especialmente (Tabla 1).

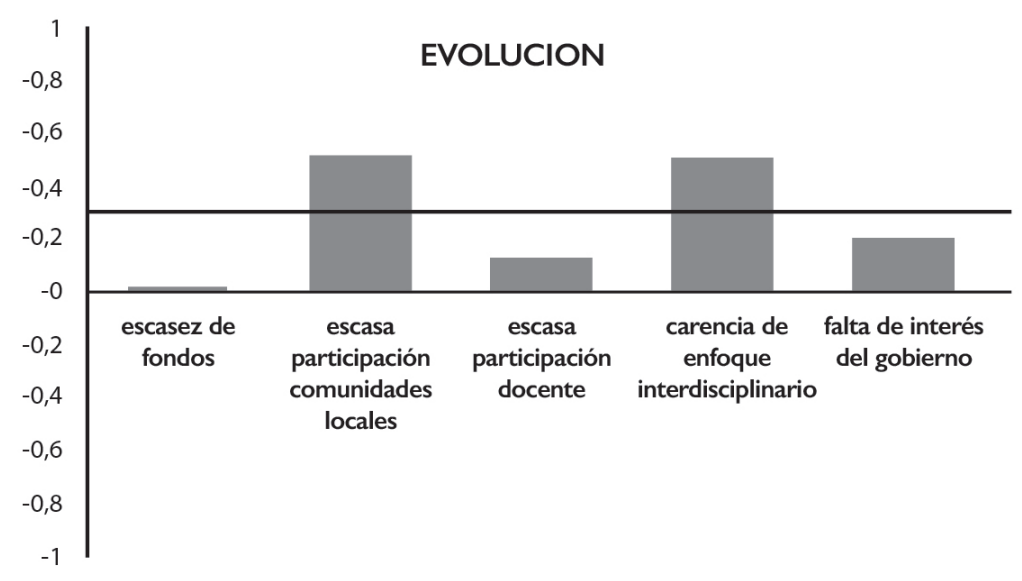

Figura 4. Evolución en los últimos 5 años de la importancia relativa de las principales dificultades para la implementación de proyectos de conservación de carnívoros terrestres en Argentina. El valor del índice puede variar entre -1, si todos los encuestados consideraran que esa dificultad se ha incrementado, y 1, si todos los encuestados opinaran que la importancia de ese factor disminuyó. La línea negra indica el valor promedio. / Variation in the last 5 years of the relative importance of the major difficulties in the implementation of of conservation projects on terrestrial carnivores in Argentina. The value of the index may vary from -1 when all interviewees consider that a given difficulty aggravated, to +1 when al interviewees agreed that a difficulty became less serious. The black line shows the average value. 


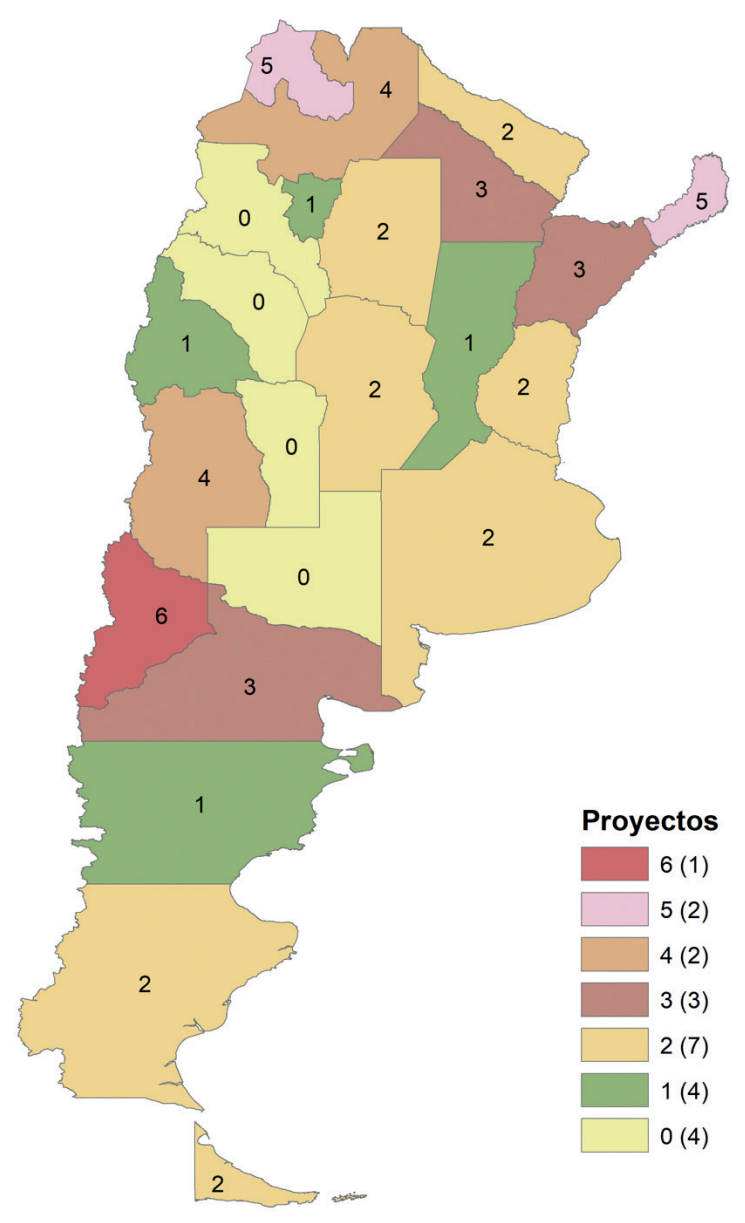

FIGURA 5. Variación espacial en el esfuerzo de conservación de carnívoros terrestres de Argentina, medido por cantidad de proyectos de conservación por provincia. Los números en paréntesis indican la cantidad de provincias por cada clase. Valores extraídos de la Apéndice II. / Spatial variation in the conservation efforts for the terrestrial carnivores in Argentina, measured as the number of conservation projects per province. The Values in brackets show the number of provinces within each category. Values were extracted from Appendix II.

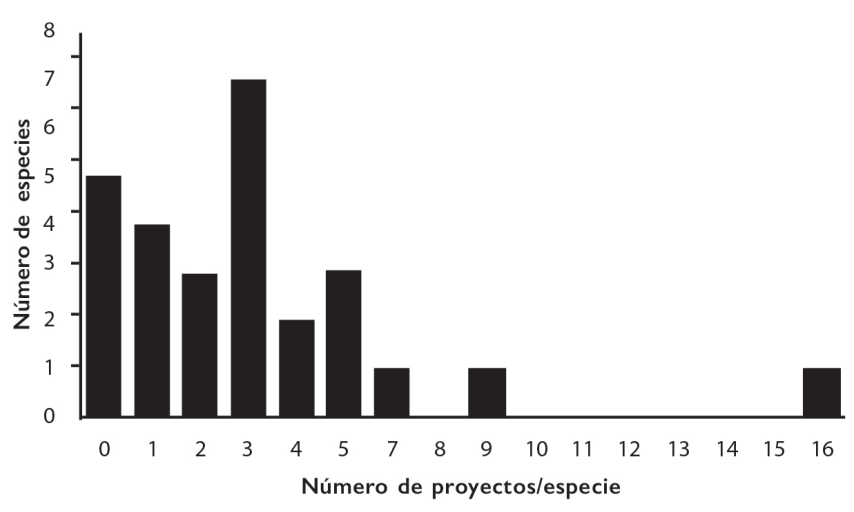

Figura 6. Esfuerzo de conservación de carnívoros terrestres de Argentina, medido por cantidad de proyectos de conservación por especie. Valores extraídos de la Apéndice II. / Conservation efforts for the terrestrial carnivores in Argentina, measured as the number of conservation projects per species. Values were extracted from Appendix II.

TABLA 1. Distribución del esfuerzo de conservación de carnívoros terrestres en Argentina, medido por el número de proyectos de conservación por especie, en función de tres clases de peso corporal. Se muestra el esfuerzo tanto para todas las especies, como aquellas indicadas como "focales" para cada proyecto listado en la Apéndice II. / Distribution of conservation effort on terrestrial carnivores in Argentina in relation to species body weight. Effort is measured as the number of conservation projects per species and is reported for all the species and for those indicated as focal species for each project listed in Appendix II.

\begin{tabular}{lcc} 
& \multicolumn{2}{c}{ N. PROYeCtos/ESPECIE } \\
\cline { 2 - 3 } Peso CORPORAL & Todas Las ESPECIES & EsPeCIES FOCALES \\
\hline Grande & 9,33 & 8,33 \\
Mediano & 2,89 & 1,11 \\
Pequeño & 2,13 & 0,67 \\
\hline
\end{tabular}




\section{DISCUSIÓN}

Las amenazas de origen antrópico a la biodiversidad se intensifican constantemente (CBD 2010; Harrop \& Pritchard 2011) y el éxito que están teniendo los proyectos de conservación con respecto a la pérdida de biodiversidad es limitado (Hayes 2006). Los problemas para la implementación de proyectos de conservación de carnívoros detectados en este estudio son comunes para los proyectos de conservación de la biodiversidad y se considera que el fracaso de estos esfuerzos se debe a una combinación de barreras sociales, científicas y políticas (Restani \& Marzluff 2002). Uno de los factores que contribuyen a esta escasez de resultados positivos es que los investigadores que se dedican a la conservación no logran transferir sus resultados a los ámbitos en los cuales se toman las decisiones (Possingham 2009; Sutherland et al. 2012; Arlettaz et al. 2010), confirmando de esta forma uno de los factores encontrados en este estudio. Otro de los problemas que limitan la eficacia de los esfuerzos de conservación es la dificultad en identificar los tópicos para los cuales es necesaria información relevante (Sutherland et al. 2012). Este trabajo se propone contribuir a reducir estas brechas y mejorar la eficacia de los esfuerzos para la conservación de los carnívoros sudamericanos.

En particular, este trabajo analizó los principales obstáculos que enfrentan los investigadores en este tipo de proyectos de conservación en Argentina y propuso medidas y líneas de acción que permitirían superarlas. Si bien no se puede excluir que el número acotado de participantes en esta revisión pueda limitar el valor de los resultados obtenidos, es preciso mencionar que los autores están involucrados en más de la mitad $(51.7 \%)$ de todos los proyectos de conservación de carnívoros que se estaban desarrollando en Argentina al momento de realizar el estudio.

El insuficiente nivel de colaboración y comunicación entre investigadores, conservacionistas, políticos y actores locales en la definición e implementación de acciones para la conservación y el uso sustentable de los recursos naturales es quizás la principal de las barreras identificadas en este trabajo. Nuestro análisis muestra claramente que la superación de este obstáculo, seguramente no limitado a la realidad argentina (Shanley \& López 2009), es una tarea que va más allá del ámbito de quienes desarrollan proyectos de conservación in-situ, involucra en forma determinante las instituciones gubernamentales y abarca todos los niveles de la sociedad. Un cambio de paradigmas y prioridades en nuestra sociedad contribuiría también a solucionar otros de los problemas identificados, favoreciendo una mayor disponibilidad de fondos para la conservación de la biodiversidad, que el sistema educativo se involucre mayormente en la conservación y que sea más fácil atraer hacia este tipo de actividades a profesionales de diferentes disciplinas.
La comparación entre las últimas dos Listas Rojas de Argentina (Díaz \& Ojeda 2000; Ojeda et al. 2012) indica que el estado de conservación de los carnívoros terrestres no ha ido mejorando en la última década. En general, las características ecológicas de los carnívoros conllevan a que su conservación no sea una tarea sencilla. Las bajas densidades poblacionales, los grandes requerimientos espaciales y sus hábitos tróficos los hacen más sensibles a las modificaciones ambientales y a las presiones antrópicas, en particular en el caso de las especies de mayor tamaño para las cuales los conflictos con las actividades ganaderas derivan en algunas de las principales causas de reducción en las poblaciones (Treves \& Karanth 2003; Woodroffe et al. 2005). Es probable que estas particularidades influyan también en los sesgos que detectamos hacia ciertas especies de carnívoros con respecto a otras y que concuerdan ampliamente con los resultados de algunas revisiones a nivel global sobre los esfuerzos de investigación (Amori \& Gippoliti 2000; Brooke et al. 2014; Zanin et al. 2015). El gran número de proyectos sobre las especies de gran tamaño corporal está posiblemente relacionado con que éstas son las que generan más conflictos con las actividades humanas o que fueron seleccionadas por ser paraguas de otras especies menores. Sin embargo, es posible que jueguen otros factores ajenos a la biología al momento de establecer las especies a las cuales dedicarse, como el atractivo para el gran público (que, a la vez, puede facilitar la recaudación de fondos; Wilson et al. 2007; Brooke et al. 2014), o consideraciones logísticas. La falta de una relación positiva entre el nivel de amenaza de las especies y el esfuerzo de investigación y conservación indica que, desafortunadamente, no siempre las especies que requieren más atención la reciben (Brooke et al. 2014). Cabe destacar que todavía tres de las nueve especies categorizadas como en peligro crítico o en peligro a nivel nacional $(P$. brasilensis, L. felina y L. guigna) a la fecha de realizar nuestra recopilación no estaban incluidas en ningún proyecto de conservación. Esto se debe, probablemente, a su rareza y las dificultades que implica trabajar con ellas en nuestro país, como demuestran los escasos resultados obtenidos sobre L. guigna por dos proyectos independientes que eligieron a esta especie como objeto de estudio y conservación en el pasado (M. Monteverde, com. pers. y M. Lucherini, datos no publicados). En forma similar al sesgo con respecto al tamaño corporal, es lógico que la falta de homogeneidad geográfica que se observa en la lista de proyectos de conservación dependa de aspectos tanto de origen ecológico como de otra naturaleza. Las tres provincias alcanzadas por el mayor número de proyectos (Neuquén, Misiones y Jujuy) ostentan una gran diversidad de ecosistemas y especies, pero en dos de ellas también se encuentran dos de los equipos de investigación con mayor trayectoria en el estudio y conservación de carnívoros terrestres y cuyos investigadores suman siete proyectos. 
Una potencial limitación relacionada con este tema y que no ha sido capturada por nuestro inventario de proyectos es la trayectoria de éstos. Nuestros análisis se basan en una lista que no discrimina entre proyectos de largo plazo, recursos relativamente amplios y otros que están en sus primeros pasos y cuentan con recursos más limitados. Finalmente, es también posible que la lista de proyectos no sea completa. Sin embargo, consideramos que un análisis más detallado de estos aspectos excede los objetivos de nuestra revisión y que estas limitaciones no afectan en forma fundamental nuestras conclusiones generales.

Independientemente de las razones que producen los sesgos en los esfuerzos de conservación que describimos, es deseable que en el futuro en Argentina los proyectos de conservación traten de establecer prioridades taxonómicas y geográficas, para enfocarse en las especies con mayor riesgo de extinción y para las cuales hay escasa información disponible, así como para ampliar las actividades hacia regiones del país que han recibido poca atención hasta el momento. Esta estrategia permitiría, además, maximizar el uso de los limitados recursos disponibles para la conservación de la biodiversidad, una reconocida necesidad a nivel global (Master 1991; Wilson et al. 2006).

A pesar de las dificultades encontradas, los resultados de nuestra revisión dan lugar a un moderado optimismo. Antes que todo, las opiniones recolectadas indican que todos los problemas identificados presentarían una tendencia a reducirse o, a lo sumo, invariada en los últimos 5 años. Más del $80 \%$ de las provincias administrativas de Argentina y más de 3 de cada 4 especies de carnívoros son foco de proyectos de conservación. Por último, si bien no hay un parámetro que permita comparar con el pasado, el número de proyectos, la cantidad de equipos de trabajo y las publicaciones que éstos producen parece en franco crecimiento en la última década.

Finalmente, cabe mencionar que esta revisión no se propuso analizar los factores próximos que amenazan las especies de carnívoros del territorio argentino sino aquellos que afectan el desempeño de los esfuerzos para su conservación que se están llevando a cabo en el país. La forma más correcta para medir el éxito de las estrategias de conservación de los carnívoros, así como de toda la biodiversidad en general, es por medio de indicadores de las tendencias poblacionales de las especies que se proponen conservar (Winterbach et al. 2013). Confiamos que la identificación de los problemas que limitan la eficacia de los esfuerzos de conservación sea el primer paso para su solución y que repercuta en una mejora del estado de las poblaciones de carnívoros.

$\mathrm{Si}$ bien Argentina no forma parte de las regiones identificadas como prioritarias para la conservación de los carnívoros a nivel global sobre la base de criterios económicos y de vulnerabilidad (Loyola et al. 2009), es evidente que tanto por la diversidad de especies de este país como por el tamaño del territorio que abarca, la conservación de las poblaciones en Argentina contribuiría en forma determinante a la de los carnívoros sudamericanos, como sugiere una reciente revisión de las prioridades a nivel global para la conservación de los félidos (Dickman et al. 2015). Por esta razón, la superación de las limitaciones identificadas en esta revisión tiene la potencialidad de mejorar el estado de conservación global de los carnívoros terrestres.

\section{ACCIONES FUTURAS}

Teniendo en cuenta los obstáculos identificados en este estudio, los participantes del Taller sugirieron las siguientes áreas de acción como prioritarias para los investigadores que se propongan una mejora en la eficacia de sus esfuerzos de conservación de carnívoros:

- Implementar proyectos de conservación a largo plazo para incrementar la posibilidad de lograr una mayor participación de las poblaciones locales, de involucrar a instituciones gubernamentales y de obtener una mayor credibilidad para el proyecto y el personal que lo lleva a cabo. Lamentablemente, los proyectos de conservación en muchos casos se llevan a cabo con fondos o con personal (por ejemplo becarios) que se supone están dedicados a la investigación. Esto implica la necesidad de obtener datos que se puedan publicar a corto plazo y no siempre de gran utilidad para la conservación a largo plazo.

- En muchas áreas los objetivos de los proyectos de conservación de carnívoros establecen conflictos culturales, porque las poblaciones locales tienen visiones diferentes sobre los carnívoros a las de los investigadores. Surge, por lo tanto, el desafío de encontrar soluciones a problemas de conservación que respeten la cultura y las creencias populares, en particular en regiones en las cuales persisten comunidades originarias. Así mismo es necesario intensificar los esfuerzos para aumentar la participación de las comunidades locales en la conservación.

- Las políticas socioeconómicas (o la falta de ellas) de las instituciones gubernamentales favorecen frecuentemente actividades productivas que provocan daños a los ecosistemas naturales. En el caso de los carnívoros, por ejemplo, pueden verse favorecidas decisiones y un manejo del ganado por parte de los productores que incrementan el potencial de los conflictos con los carnívoros. El desafío, en este caso, consiste en apoyar la identificación e implementación por parte de las entidades gubernamentales de herramientas de manejo alternativas que permitan mitigar los conflictos.

- Fortalecer las actividades de extensión a la sociedad, que logren implantar un debate amplio y abierto sobre la conservación de los carnívoros como componentes claves de la biodiversidad. El objetivo de esta línea de acción es el de favorecer la creación de una masa crítica de ciudadanos a favor de la conservación, que influya sobre el gobierno en los distintos niveles e instancias políticas. 
- Enfrentar los problemas de pérdida y fragmentación de hábitat a una escala que permita tomar en consideración el flujo génico entre las poblaciones de las especies que se quiere conservar y los efectos sobre las dinámicas meta-poblacionales y generar estrategias de conservación comprensivas. Para lograr este tipo de estrategias sería de gran ayuda también una planificación por parte de los gobiernos que apoye este tipo de proyectos, así como programas que favorezcan la conectividad ambiental y la conservación de endemismos, de poblaciones viables y de la riqueza específica de los ecosistemas.

- Buscar y favorecer el diálogo y el trabajo en conjunto con las instituciones del gobierno. En particular, es menester de los investigadores cumplir con los compromisos de informar a estas instituciones los resultados de las investigaciones y las propuestas de manejo/conservación realizadas, para brindar herramientas que permitan trabajar en dichas temáticas y favorecer políticas apropiadas para los problemas a enfrentar.

- Favorecer el cumplimiento por parte de las instituciones que reúnen a los investigadores que se ocupan de conservación (SAREM, CONICET, ASAE, entre otras) de un rol de nexo más efectivo entre los proyectos de conservación y los gobiernos. Si bien esta no debe ser tomada como una oportunidad para descargar responsabilidades de parte de los integrantes de proyectos de conservación, el apoyo de estas instituciones podría ayudar a superar algunos de los obstáculos que se identificaron.

- Apoyar la generación por parte del gobierno de puestos rentados de Educadores Ambientales o Agentes de Conservación, que sean ocupados por personas preparadas profesionalmente para esta función, e implementar acciones para la articulación de las actividades de estos profesionales y las necesidades detectadas por los investigadores que trabajan en proyectos de conservación. Esto permitiría una mejora en la calidad de las acciones de difusión y participación comunitaria y, al mismo tiempo, dejaría más espacio a los investigadores para contribuir a la conservación realizando las tareas para las cuales están mejor capacitados.

- Favorecer una mayor presencia de los conceptos relacionados a la conservación de la biodiversidad en general, y de los carnívoros en particular, en los contenidos curriculares de las escuelas. El personal docente de los establecimientos educativos, en particular aquellos ubicados en zonas rurales, debería tener estímulo y apoyo de los ministerios de educación para poder capacitarse en temas de conservación y para llevar a cabo proyectos relacionados con la conservación con sus alumnos.

- Promover una política de conservación a nivel nacional, que, en el caso de los carnívoros, identifique prioridades, así como herramientas y objetivos focales, y favorezca la implementación de proyectos que se encarguen de ellas.

- Enfocar las actividades futuras de estudio y conservación de los carnívoros hacia las especies y las regiones del país que no han sido incluidas en los proyectos implementados hasta la fecha.

\section{AGRADECIMIENTOS}

Agradecemos a Tadeu de Oliveira, Claudia Manfredi, Emma Casanave, Estela Luengos Vidal, y a todo el comité organizador de las Jornadas de XXIII Jornadas de la SAREM. K. DeMatteo, M. Di Bitetti, A. Paviolo, A. Brown, N. Lodeiro Ocampo, I. Jiménez Pérez, A. Novaro, P. Perovic, A. Valenzuela, V. Quiroga y M.J. Merino brindaron su tiempo y la información necesaria para completar la lista de proyectos. La Agencia Nacional de Promoción Científica y Tecnológica, la Secretaría General de Ciencia y Tecnología de la Universidad Nacional del Sur y la Cooperativa Obrera apoyaron financieramente la organización de las Jornadas.

\section{REFERENCIAS}

Amori, G., Gippoliti, S. 2000. What do mammalogists want to save? Ten years of mammalian conservation biology. Biodiversity and Conservation 9: 785-793.

Arlettaz, R., Schaub, M., Fournier, J., Reichlin T.S., Sierro, A., Watson, J.E., BRAUNISCH, V. 2010. From publications to public actions: when conservation biologists bridge the gap between research and implementation. BioScience 60: $835-842$.

BAILEY, K.D. 1982. Methods of social research. The Free Press, New York.

Boitani, L., Sinibaldi, I., Corsi, F., De Biase, A., D’inzillo Carranza, I., Ravagli, M., Reggiani, G., Rondinini, C., Trapanese, P. 2007. Distribution of medium- to largesized African mammals based on habitat suitability models. Biodiversity and Conservation 17: 605-621.

Brooke, Z.M., Bielby, J., Nambiar, K., Carbone, C. 2014. Correlates of research effort in carnivores: body size, range size and diet matter. PloS ONE 9(4): e93195.

Canevari, M., Vaccaro, O. 2007. Guía de mamíferos del sur de América del Sur. Editorial L.O.L.A., Buenos Aires.

CBD (Convention on Biological Diversity). 2010. Secretariat of the Convention on Biological Diversity. Global Biodiversity Outlook 3, Montréal.

Chebez, J.C., Pardiñas U., Teta, P. 2014. Mamíferos terrestres de la Patagonia, sur de Argentina y Chile. Vazquez Mazzini, Buenos Aires.

Clevenger, A.P., Wierzchowski, J., Chruszcz, B., Gunson, K. 2002. GIS-generated, expert-based models for identifying wildlife habitat linkages and planning mitigation passages. Conservation Biology 16: 503-514.

Díaz, G.B., OJedA, R.A. (eds.) 2000. Libro rojo de mamíferos amenazados de la Argentina. Sociedad Argentina para el Estudio de Mamíferos, Buenos Aires.

Dickman, A.J., Hinks, A.E., Macdonald, E.A., Burnham. D., Macdonald, D.W. 2015. Priorities for global felid conservation. Conservation Biology 29: 854-864.

GinsBerg, J.R. 2001. Setting priorities for carnivore conservation: 
What makes carnivores different? En: Gittleman, J.L., Funk, S.M., Macdonald, D., Wayne, R.K. (Eds) Carnivore Conservation: 498-523. Cambridge University Press, Cambridge.

Gusset, M., FA, F.A., Sutherland, W.J. 2014. A horizon scan for species conservation by zoos and aquariums. Zoo Biology 33: 375-380.

Harrop, S.R., Pritchard, D.J. 2011. A hard instrument goes soft: the implications of the Convention on Biological Diversity's current trajectory. Global Environmental Change 21: 474-480.

HAYES, T.M. 2006. Parks, people, and forest protection: an institutional assessment of the effectiveness of protected areas. World Development 34: 2064-2075.

Karanth, K.U., Chellam, R. 2009. Carnivore conservation at the crossroads. Oryx 43:1-2.

Loyola, R.D., Oliveira-Santos, L.G.R., Almeida-Neto, M., Nogueira, D.M., Kubota, U., Diniz-Filho, J.A.F., LEWINSOHN, T.M. 2009. Integrating economic costs and biological traits into global conservation priorities for carnivores. PloS ONE 4(8): e6807.

Lucherini, M., Soler, L., LuEngos VidAL, E.M. 2004. A preliminary revision of knowledge status of felids in Argentina. Mastozoología Neotropical 11: 7-17.

Martin, T.G., Burgman, M.A., Fidler, F., Kuhnert, P.M., LowChoy, S., Mcbride, M., Mengersen, K. 2012. Eliciting expert knowledge in conservation science. Conservation Biology 26: 29-38.

Master, L.L. 1991. Assessing threats and setting priorities for conservation. Conservation Biology 5: 559-563.

Metrick, A., Weitzman, M.L. 1998. Conflicts and choices in biodiversity preservation. Journal of Economic Perspectives 12: 21-34.

Ojeda, R.A., Chillo, V., Díaz, G. 2012. Libro Rojo de los Mamíferos Amenazados de la Argentina. Sociedad Argentina para el Estudio de los Mamíferos (SAREM), Buenos Aires.

Possingham, H. 2009. Dealing with 'The great divide'. Decision Point 28: 1-12.

Regan, T.J., Master, L.L., Hammerson, G.A. 2004. Capturing expert knowledge for threatened species assessments: a case study using NatureServe conservation status ranks. Acta Oecologica 26: 95-107.

Restani, M., Marzluff, J.M. 2002. Funding extinction? Biological needs and political realities in the allocation of resources

\section{APÉNDICE I}

Como se menciona en el texto, este trabajo es el fruto del taller "Análisis de estrategias para la conservación de carnívoros en Latinoamérica" realizado en el marco de las XXIII Jornadas de la Sociedad Argentina para el Estudio de los Mamíferos, que se llevaron a cabo en la ciudad de Bahía Blanca, provincia de Buenos Aires, del 9 al 12 de noviembre de 2010.

Los coordinadores del Taller (Mauro Lucherini, Claudia to endangered species recovery. BioScience 52: 169-177.

Ripple, W.J., Estes, J.A., Beschta, R.L., Wilmers, C.C., Ritchie, E.G., Hebblewhite, M., Berger, J., Elmhagen, B., Letnic, M., Nelson, M.P., Schmitz, O.J., Smith, D.W., Wallach, A.D., Wirsing, A.J. 2014. Status and Ecological Effects of the World's Largest Carnivores. Science 343: 1241484.

Sergio, F., Caro, T., Brown, D., Clucas, B., Hunter, J., Ketchum, J., Mchugh, K., Hiraldo, F. 2008. Top predators as conservation tools: ecological rationale, assumptions, and efficacy. Annual Review of Ecology, Evolution, and Systematics 39: 1-19.

Shanley, P., López, C. 2009. Out of the loop: why research rarely reaches policy makers and the public and what can be done. Biotropica 41: 535-544.

Sutherland, W.J., Pullin, A.S., Dolman, P.M., Knight, T.M. 2004. The need for evidence-based conservation. Trends in Ecology \& Evolution 19: 305-308.

Sutherland, W.J., Allison, H., Aveling, R., Bainbridge, I.P., Bennun, L., Bullock, D.J., Clements, A., Crick, H.Q.P., Gibbons, D.W., Smith, S., Rands, M.R.W., Rose, P., Scharlemann, J.P.W., Warren, M.S. 2012. Enhancing the value of horizon scanning through collaborative review. Oryx 46: 368-374.

Treves, A., Karanth, K.U. 2003. Human-carnivore conflict and perspectives on carnivore management worldwide. Conservation Biology 17: 1491-1499.

Wilson, J.R.U., Proches, S., Braschler, B., Dixon, E.S., Richardson, D.M. 2007. The (bio)diversity of science reflects the interests of society. Frontiers in Ecology and Environment 5: 409-414.

Wilson, K.A., Mcbride, M.F., Bode. M., Possingham, H.P. 2006. Prioritizing global conservation efforts. Nature 440: 337340.

Winterbach, H.E.K., Winterbach, C.W., Somers, M.J., Hayward, M.W. 2013. Key factors and related principles in the conservation of large African carnivores. Mammal Review 43: 89-110.

Woodroffe, R., Thirgood, S., Rabinowitz, A. 2005. The impact of human-wildlife conflict on natural systems. En: Woodroffe, R., Thirgood, S, Rabinowitz, A.R. eds. People and Wildlife: Conflict or Coexistence?: 1-12. Cambridge University Press, Cambridge.

Zanin, M., Palomares, F., Brito, D. 2015. What we (don't) know about the effects of habitat loss and fragmentation on felids. Oryx 49: 96-106.

Manfredi y Juan I. Reppucci, del Grupo de Ecología Comportamental de Mamíferos - Laboratorio de Fisiología Animal, Instituto de Ciencias Biológicas y Biomédicas del Sur, Depto. de Biología Bioquímica y Farmacia, Universidad Nacional del Sur - CONICET) invitaron a otros investigadores conocidos por sus proyectos de estudio y conservación de carnívoros terrestres de Argentina y otros países latinoamericanos, logrando la participación de los autores del presente trabajo y de Tadeu de Oliveira, un investigador de la Universidade Estadual do Maranhão, Brasil. 


\section{APÉNDICE II}

Información básica (título, ubicación geográfica, responsables, especies focales y secundarias involucradas) de todos los proyectos de conservación in-situ de carnívoros terrestres actualmente en curso en Argentina. *: especies focales para cada uno de los proyectos listados. / Basic information (Title, geographic location, Principal Investigators, focal and secondary species) of all the in-situ conservation projects on terrestrial carnivores currently executed in Argentina. *: focal species for each listed project.

\begin{tabular}{|c|c|c|c|}
\hline Proyecto & InVESTIGADORES PRINCIPALES & Provincias & $\begin{array}{l}\text { ESPECIES DE } \\
\text { CARNÍVOROS }\end{array}$ \\
\hline $\begin{array}{l}\text { Conservación de los carnívoros del nordeste } \\
\text { argentino mediante la participación de los } \\
\text { pobladores locales }\end{array}$ & $\begin{array}{l}\text { Lucia Soler } \\
\text { María Jesús Palacios González } \\
\text { Karina Iaconis }\end{array}$ & $\begin{array}{l}\text { Corrientes } \\
\text { Chaco }\end{array}$ & $\begin{array}{l}\text { C. brachyurus } \\
\text { P. gymnocercus } \\
\text { C. thous } \\
\text { L. longicaudis } \\
\text { P. cancrivorus } \\
\text { L. geoffroyi } \\
\text { P. concolor } \\
\text { P. yaguaroundi }\end{array}$ \\
\hline Proyecto Madriguera & $\begin{array}{l}\text { Lucia Soler } \\
\text { Fernando Del Moral Sachetti }\end{array}$ & Chubut & $\begin{array}{l}\text { P. concolor* } \\
\text { P. culpaeus }\end{array}$ \\
\hline Proyecto de conservación de aguará guazú & $\begin{array}{l}\text { Paula González Ciccia } \\
\text { Guillermo Delfino } \\
\text { Andrea Pierini }\end{array}$ & Córdoba & C. brachyurus* \\
\hline $\begin{array}{l}\text { Plan de conservación del aguará guazú en } \\
\text { Argentina }\end{array}$ & $\begin{array}{l}\text { Lucia Soler } \\
\text { Marcela Orozco } \\
\text { Paula González Ciccia }\end{array}$ & $\begin{array}{l}\text { Formosa } \\
\text { Corrientes } \\
\text { Chaco } \\
\text { Misiones } \\
\text { Entre Ríos } \\
\text { Santa Fe } \\
\text { Santiago del } \\
\text { Estero } \\
\text { Córdoba }\end{array}$ & C. brachyurus* \\
\hline $\begin{array}{l}\text { Implementación de manejo de ganado bovino } \\
\text { como herramienta para la conservación a largo } \\
\text { plazo del jaguar en las selvas de Yungas del } \\
\text { noroeste de Argentina }\end{array}$ & $\begin{array}{l}\text { Pablo Perovic } \\
\text { Claudio Sillero } \\
\text { Juan Arrabal }\end{array}$ & $\begin{array}{l}\text { Jujuy } \\
\text { Salta }\end{array}$ & $\begin{array}{l}\text { P. onca* } \\
\text { P. concolor* }\end{array}$ \\
\hline $\begin{array}{l}\text { Estimación poblacional de yaguareté en las } \\
\text { yungas del noroeste de Argentina }\end{array}$ & $\begin{array}{l}\text { Pablo Perovic } \\
\text { Claudio Sillero }\end{array}$ & $\begin{array}{l}\text { Jujuy } \\
\text { Salta }\end{array}$ & P. onca* \\
\hline $\begin{array}{l}\text { Conservación del gato andino patagónico. } \\
\text { Mitigación de conflictos y fortalecimiento de } \\
\text { capacidades }\end{array}$ & $\begin{array}{l}\text { Andrés Novaro } \\
\text { Susan Walker } \\
\text { Carolina Marull } \\
\text { Lara Heidel } \\
\text { María José Bolgeri } \\
\text { Martín Funes }\end{array}$ & $\begin{array}{l}\text { Neuquén } \\
\text { Mendoza }\end{array}$ & L. jacobita* \\
\hline El Alma de los Andes & $\begin{array}{l}\text { Mauro Lucherini } \\
\text { Juan Reppucci } \\
\text { Cintia Tellaeche }\end{array}$ & Jujuy & $\begin{array}{l}\text { L. jacobita* } \\
\text { L. colocolo } \\
\text { P. culpaeus } \\
\text { P. concolor }\end{array}$ \\
\hline $\begin{array}{l}\text { Proyecto yaguareté: uniendo esfuerzos para } \\
\text { conservar la poblaciones de yaguareté del Bosque } \\
\text { Atlántico }\end{array}$ & $\begin{array}{l}\text { Agustín Paviolo } \\
\text { Carlos De Angelo } \\
\text { Mario Di Bitetti } \\
\text { Verónica Quiroga y colaboradores }\end{array}$ & Misiones & $\begin{array}{l}\text { P. onca* } \\
\text { P. concolor* }\end{array}$ \\
\hline
\end{tabular}




\begin{tabular}{|c|c|c|c|}
\hline Proyecto & InVESTIGADORES PRINCIPALES & Provincias & $\begin{array}{l}\text { ESPECIES DE } \\
\text { CARNÍVOROS }\end{array}$ \\
\hline $\begin{array}{l}\text { Impacto de los carnívoros silvestres sobre las } \\
\text { actividades productivas ganaderas en el sudoeste } \\
\text { bonaerense: caracterización y mitigación de } \\
\text { conflictos }\end{array}$ & $\begin{array}{l}\text { Mauro Lucherini } \\
\text { Estela Luengos Vidal } \\
\text { Nicolás Caruso } \\
\text { Diego Castillo } \\
\text { María Guerisoli } \\
\text { Emma Caasanave }\end{array}$ & Buenos Aires & $\begin{array}{l}\text { P. concolor* } \\
\text { P. gymnocercus } * \\
\text { L. geoffroyi } \\
\text { C. chinga } \\
\text { L. colocolo } \\
\text { G. cuja }\end{array}$ \\
\hline $\begin{array}{l}\text { Variables ambientales y antrópicas que afectan la } \\
\text { distribución del gato andino en Patagonia norte }\end{array}$ & $\begin{array}{l}\text { Rocío Palacios } \\
\text { Susan Walker } \\
\text { Raquel Gleiser }\end{array}$ & $\begin{array}{l}\text { Neuquén } \\
\text { Mendoza }\end{array}$ & $\begin{array}{l}\text { L. jacobita* } \\
\text { L. colocolo } \\
\text { P. culpaeus } \\
\text { P. griseus } \\
\text { P. concolor }\end{array}$ \\
\hline $\begin{array}{l}\text { Programa EduGat: educación para la conserva- } \\
\text { ción del gato andino y su hábitat }\end{array}$ & $\begin{array}{l}\text { María José Merino } \\
\text { Mauro Lucherini }\end{array}$ & $\begin{array}{l}\text { Neuquén } \\
\text { Jujuy }\end{array}$ & $\begin{array}{l}\text { L. jacobita* } \\
\text { L. colocolo }\end{array}$ \\
\hline $\begin{array}{l}\text { Conservación de mamíferos carnívoros en Pata- } \\
\text { gonia: neofobia y aversión condicionada al sabor } \\
\text { como herramientas para un control selectivo y } \\
\text { protección de especies no perjudiciales }\end{array}$ & $\begin{array}{l}\text { Alejandro Travaini } \\
\text { Sonia Zapata } \\
\text { Diego Procopio } \\
\text { Sigrid Nielsen }\end{array}$ & Santa Cruz & $\begin{array}{l}\text { P. culpaeus* } \\
\text { P. griseus } *\end{array}$ \\
\hline $\begin{array}{l}\text { Control selectivo de predadores: una experiencia } \\
\text { de investigación, transferencia y mejora del } \\
\text { sistema socioeconómico rural basado en prácticas } \\
\text { ganaderas sustentables y conservación de la } \\
\text { biodiversidad }\end{array}$ & $\begin{array}{l}\text { Alejandro Travaini } \\
\text { Sonia Zapata } \\
\text { Diego Procopio }\end{array}$ & Santa Cruz & $\begin{array}{l}\text { P. culpaeus* } \\
\text { P. griseus* } \\
\text { P. concolor* }\end{array}$ \\
\hline Ecología y conservación del huillín & Alejandro Valenzuela & $\begin{array}{l}\text { Tierra del Fuego } \\
\text { Río Negro }\end{array}$ & L. provocax* \\
\hline $\begin{array}{l}\text { Plan de monitoreo de la población marina de } \\
\text { huillín. Parque Nacional Tierra del Fuego }\end{array}$ & $\begin{array}{l}\text { Alejandro Valenzuela } \\
\text { Emilce Gallo } \\
\text { Guillermina Massaccesi } \\
\text { Laura Malmierca }\end{array}$ & Tierra del Fuego & L. provocax* \\
\hline $\begin{array}{l}\text { Conservación de la población dulceacuícola } \\
\text { de huillín en Argentina: plan de monitoreo del } \\
\text { Parque Nacional Nahuel Huapi }\end{array}$ & $\begin{array}{l}\text { Claudio Chehèbar } \\
\text { Carla Pozzi } \\
\text { Susana Seijas }\end{array}$ & $\begin{array}{l}\text { Neuquén } \\
\text { Río Negro }\end{array}$ & L. provocax* \\
\hline $\begin{array}{l}\text { Reducción de conflictos entre productores } \\
\text { ganaderos y carnívoros en Patagonia }\end{array}$ & $\begin{array}{l}\text { Andrés Novaro } \\
\text { Susan Walker } \\
\text { Carolina Marull } \\
\text { María José Bolgeri } \\
\text { Martín Funes }\end{array}$ & $\begin{array}{l}\text { Neuquén } \\
\text { Mendoza }\end{array}$ & $\begin{array}{l}\text { L. jacobita* } \\
\text { P. concolor* } \\
\text { P. gymnocercus } \\
\text { L. geoffroyi } \\
\text { L. colocolo } \\
\text { P. culpaeus }\end{array}$ \\
\hline Interacción puma-guanaco en Patagonia norte & $\begin{array}{l}\text { Andrés Novaro } \\
\text { Mariel Ruiz Blanco } \\
\text { María José Bolgeri } \\
\text { Susan Walker } \\
\text { Martín Funes } \\
\text { Emiliano Donadio }\end{array}$ & $\begin{array}{l}\text { Mendoza } \\
\text { Neuquén }\end{array}$ & P. concolor* \\
\hline $\begin{array}{l}\text { Interacciones depredador-presa en sistemas } \\
\text { semiáridos de Argentina: efectos directos e } \\
\text { indirectos sobre poblaciones y comunidades }\end{array}$ & Emiliano Donadio & San Juan & P. concolor* \\
\hline $\begin{array}{l}\text { Evaluación de refugios y corredores para } \\
\text { vertebrados en paisajes forestales del bajo Delta } \\
\text { del Paraná }\end{array}$ & $\begin{array}{l}\text { Natalia Fracassi } \\
\text { Javier Pereira }\end{array}$ & $\begin{array}{l}\text { Buenos Aires } \\
\text { Entre Ríos }\end{array}$ & $\begin{array}{l}\text { L. geoffroyi* } \\
\text { C. thous } \\
\text { G. cuja } \\
\text { L. longicaudis }\end{array}$ \\
\hline
\end{tabular}


Esfuerzos de conservación de carnívoros en Argentina: LuCHERINI, M. ET AL.

\begin{tabular}{|c|c|c|c|}
\hline PRoyecto & InVESTIGADORES PRINCIPALES & Provincias & $\begin{array}{l}\text { EsPECIES DE } \\
\text { CARNÍVOROS }\end{array}$ \\
\hline $\begin{array}{l}\text { Influencia de factores socio-económicos y } \\
\text { ambientales sobre el conflicto carnívoros- } \\
\text { ganadería }\end{array}$ & $\begin{array}{l}\text { Pablo Gáspero } \\
\text { Valeria Fernandez Arhex } \\
\text { Javier Pereira }\end{array}$ & $\begin{array}{l}\text { Río Negro } \\
\text { Neuquén }\end{array}$ & $\begin{array}{l}\text { P. concolor* } \\
\text { P. culpaeus* }\end{array}$ \\
\hline $\begin{array}{l}\text { Influencia de las actividades humanas sobre la } \\
\text { ecología y la conservación del yaguareté en las } \\
\text { Yungas de la Argentina }\end{array}$ & $\begin{array}{l}\text { Nicolás Lodeiro Ocampo } \\
\text { Fernando Falke } \\
\text { Mariela Gantchoff }\end{array}$ & Salta & P. onca* \\
\hline $\begin{array}{l}\text { Programa de mitigación del impacto sobre la } \\
\text { fauna silvestre producido por automotores en } \\
\text { rutas de la provincia de Misiones }\end{array}$ & Nicolás Lodeiro Ocampo & Misiones & $\begin{array}{l}\text { P. onca* } \\
\text { P. concolor* } \\
\text { L. pardalis* }\end{array}$ \\
\hline $\begin{array}{l}\text { Proyecto de cría experimental y de promoción } \\
\text { del yaguareté en los Esteros de Ibera }\end{array}$ & $\begin{array}{l}\text { Gustavo Solís } \\
\text { Ignacio Jiménez Pérez } \\
\text { Sofía Heinonen } \\
\text { Jorge Peña } \\
\text { Karina Spøerring }\end{array}$ & Corrientes & P. onca* \\
\hline $\begin{array}{l}\text { Distribución, abundancia e interacciones } \\
\text { ecológicas de los felinos medianos y pequeños } \\
\text { del Bosque Atlántico del Alto Paraná de la } \\
\text { provincia de Misiones }\end{array}$ & $\begin{array}{l}\text { María Paula Cruz } \\
\text { Mario Di Bitetti } \\
\text { Agustín Paviolo } \\
\text { Karen DeMatteo }\end{array}$ & Misiones & $\begin{array}{l}\text { L. wiedii* } \\
\text { L. } \text { tigrinus }^{*} \\
\text { L. pardalis* } \\
\text { P. yagouaroundi }\end{array}$ \\
\hline $\begin{array}{l}\text { Identificación de corredores de hábitat que } \\
\text { minimicen los conflictos humanos-vida silvestre } \\
\text { y maximicen la movilidad de especies por el } \\
\text { paisaje del Bosque Atlántico interior }\end{array}$ & $\begin{array}{l}\text { Karen DeMatteo } \\
\text { Miguel Rinas } \\
\text { Carina Argüelles } \\
\text { Mario Di Bitetti }\end{array}$ & Misiones & $\begin{array}{l}\text { S. venaticus* } \\
\text { P. onca* } \\
\text { P. concolor* } \\
\text { L. pardalis } \\
\text { L. tigrinus }\end{array}$ \\
\hline $\begin{array}{l}\text { Diversidad de mamíferos en paisajes productivos } \\
\text { protegidos de las Yungas argentinas: puesta en } \\
\text { valor y gestión para su conservación }\end{array}$ & $\begin{array}{l}\text { Sebastián Albanesi } \\
\text { Alejandro Brown } \\
\text { Mario Di Bitetti }\end{array}$ & $\begin{array}{l}\text { Jujuy } \\
\text { Salta } \\
\text { Tucumán }\end{array}$ & $\begin{array}{l}\text { P. onca* } \\
\text { P. concolor* } \\
\text { P. yagouaroundi } \\
\text { L. pardalis } \\
\text { L. wiedii } \\
\text { L. geoffroyi } \\
\text { L. tigrinus } \\
\text { N. nasua } \\
\text { P. cancrivorus } \\
\text { E. barbara } \\
\text { G. cuja } \\
\text { C. thous } \\
\text { P. gymnocercus }\end{array}$ \\
\hline $\begin{array}{l}\text { Ecología y conservación del yaguareté y el puma } \\
\text { en el Chaco semiárido de Argentina }\end{array}$ & Verónica Quiroga & $\begin{array}{l}\text { Santiago del } \\
\text { Estero } \\
\text { Chaco } \\
\text { Formosa }\end{array}$ & $\begin{array}{l}\text { P. onca* } \\
\text { P. concolor* }\end{array}$ \\
\hline
\end{tabular}

Recibido: 27.05.2018

Aceptado: 10.06.2018 